\title{
Identification of copy number variations using high density whole-genome single nucleotide polymorphism markers in Chinese Dongxiang spotted pigs
}

\author{
Chengbin Wang ${ }^{1, a}$, Hao Chen ${ }^{1, a}$, Xiaopeng Wang ${ }^{1}$, Zhongping $\mathrm{Wu}^{1}$, Weiwei Liu ${ }^{1}$, \\ Yuanmei Guo ${ }^{1}$, Jun Ren ${ }^{1}$, and Nengshui Ding ${ }^{1, *}$
}

\author{
* Corresponding Author: Nengshui Ding \\ Tel: +86-791-83813080, Fax: +86-791-83900189, \\ E-mail: 13631698@qq.com \\ 1 State Key Laboratory of Pig Genetic Improvement and \\ Production Technology, Jiangxi Agricultural University, \\ Nanchang 330045, China \\ a These authors contribute equally to this study. \\ ORCID \\ Chengbin Wang \\ https://orcid.org/0000-0002-5156-9067 \\ Hao Chen \\ https://orcid.org/0000-0002-5210-8924 \\ Xiaopeng Wang \\ https://orcid.org/0000-0002-6608-2892 \\ Zhongping Wu \\ https://orcid.org/0000-0001-5246-5190 \\ Weiwei Liu \\ https://orcid.org/0000-0002-1302-0209 \\ Yuanmei Guo \\ https://orcid.org/0000-0003-0381-9647 \\ Jun Ren \\ https://orcid.org/0000-0001-6664-3998 \\ Nengshui Ding \\ https://orcid.org/0000-0002-1185-5528
}

Submitted Sept 12, 2018; Revised Nov 9, 2018; Accepted Jan 8, 2019
Objective: Copy number variations (CNVs) are a major source of genetic diversity complementary to single nucleotide polymorphism (SNP) in animals. The aim of the study was to perform a comprehensive genomic analysis of CNVs based on high density whole-genome SNP markers in Chinese Dongxiang spotted pigs.

Methods: We used customized Affymetrix Axiom Pig1.4M array plates containing 1.4 million SNPs and the PennCNV algorithm to identify porcine CNVs on autosomes in Chinese Dongxiang spotted pigs. Then, the next generation sequence data was used to confirm the detected CNVs. Next, functional analysis was performed for gene contents in copy number variation regions (CNVRs). In addition, we compared the identified CNVRs with those reported ones and quantitative trait loci (QTL) in the pig QTL database.

Results: We identified 871 putative CNVs belonging to 2,221 CNVRs on 17 autosomes. We further discarded CNVRs that were detected only in one individual, leaving us 166 CNVRs in total. The $166 \mathrm{CNVRs}$ ranged from $2.89 \mathrm{~kb}$ to $617.53 \mathrm{~kb}$ with a mean value of $93.65 \mathrm{~kb}$ and a genome coverage of $15.55 \mathrm{Mb}$, corresponding to $0.58 \%$ of the pig genome. A total of $119(71.69 \%)$ of the identified CNVRs were confirmed by next generation sequence data. Moreover, functional annotation showed that these CNVRs are involved in a variety of molecular functions. More than half $(56.63 \%)$ of the CNVRs $(n=94)$ have been reported in previous studies, while 72 CNVRs are reported for the first time. In addition, 162 (97.59\%) CNVRs were found to overlap with 2,765 previously reported QTLs affecting 378 phenotypic traits.

Conclusion: The findings improve the catalog of pig CNVs and provide insights and novel molecular markers for further genetic analyses of Chinese indigenous pigs.

Keywords: Copy Number Variation; Pig1.4M Array Plate; Next Generation Sequence; Complex Traits; Chinese Indigenous Pigs

\section{INTRODUCTION}

Copy number variations (CNVs) refer to the insertion, deletion and duplication of DNA segments of $1 \mathrm{~kb}$ or larger with variable copy number compared with the reference genome. Since the milestone work by Iafrate et al [1] and Sebat et al [2] that first reported CNVs in the human genome, thousands of CNVs have been detected across the human genome. It has been reported that $\mathrm{CNVs}$ account for $3.7 \%, 4.6 \%, 4.2 \%, 1.4 \%, 3.6 \%$, and $5.8 \%$ of human, cattle, dog, rat, horse, and pig assembled genomes, respectively [3-8].

A list of CNVs are known to affect phenotypic traits in humans [3]. In livestock, CNVs have also been repeatedly shown to cause phenotypic variations. For instance, the dupli- 
cation of the proto-oncogene receptor tyrosine kinase (KIT) gene is responsible for the white coat phenotype in pigs [9]. The copy number alteration in intron 1 of the sex determining region Y-Box (SOX5) gene contributes to the pea-comb phenotype in chickens [10]. A 4.6-kb duplication in intron 6 of the syntaxin 17 gene causes hair graying and melanoma in horses [11], and CNV and missense mutations of the agouti signaling protein gene lead to different coat colors in goats [12].

Currently, CNVs can be identified using three different approaches, including single nucleotide polymorphism (SNP) genotyping array, comparative genomic hybridization and next-generation sequencing [13]. Of these three approaches, SNP genotyping array has been popular in large-scale CNV surveys due to it relatively lower cost [14]. In pigs, Fadista et al [15] first reported 37 copy number variation regions (CNVRs) that were identified in 12 unrelated Duroc pigs using the comparative genomic hybridization method. Then, Ramayo-Caldas et al [16] detected 49 CNVRs in 55 animals belonging to several generations of the Iberian boars crossing with Landrace sows using Porcine SNP60K BeadChip array. Afterwards, a number of CNVs were inferred from wholegenome $60 \mathrm{~K}$ SNP data of diverse pig breeds $[8,14,17]$.

Dongxiang spotted pigs, a representative Chinese local pig breed, was originally distributed in Dongxiang county, Jiangxi province of China. Dongxiang spotted pigs are well adapted to local environments and roughage feed occurring in the middle and lower area of the Yangtze River. This breed is also known for strong disease resistance and desirable meat quality, providing a valuable genetic resource for further genetic improvement of commercial breeds in Chinese pig industry [18]. We herein used a customized Affymetrix Axiom Pig1.4M array plate containing 1.4 million SNPs and the PennCNV algorithm to identify porcine autosomal CNVs in Chinese Dongxiang spotted pigs, aiming to improve the catalog of pig $\mathrm{CNV}$ s and facilitate the identification of trait-related CNVs for further selective breeding of pig breeds.

\section{MATERIALS AND METHODS}

\section{Ethics statements}

All animal work was carried out according to the approved guidelines established by the Ministry of Agriculture of China. The Ethics Committee of Jiangxi Agricultural University specifically approved this study.

\section{Experimental animals}

Samples were collected from ear tissues of 96 Dongxiang spotted pigs as described in our previous report [19]. Briefly, these 96 pigs were sampled from the nucleus population of Dongxiang spotted pigs in a national conservation farm in Dongxiang county, Jiangxi Province, China. The farm is the only state- supported farm specifically for the conservation of Dongxiang spotted pigs. The nucleus population currently comprises $\sim 120$ sows and 15 boars and is managed to maintain its genetic diversity without intensive selection for any particular traits.

\section{Genotyping and quality control}

Genomic DNA from the 96 individuals was extracted from ear tissue using a standard phenol/chloroform method [20]. DNA quality was measured by spectrophotometry and agarose gel electrophoresis and was finally diluted to a concentration of $50 \mathrm{ng} / \mu \mathrm{L}$. All 96 pigs were genotyped with a customized Affymetrix Axiom Pig1.4M array plate as previously described [19]. Briefly, a total of $1,362,630$ SNPs were successfully genotyped for these 96 pigs. Before CNV calling, we first performed a quality control by Plink v1.07 [21]. After quality control procedures, one individual was removed due to its missing genotype date rate $(>0.1), 678$ SNPs were discarded due to Hardy-Weinberg exact test, and 85,205 and 711,888 SNPs were discarded due to their genotype call rates $(<0.90)$ and minor allele frequencies $(<0.05)$, respectively. A final set of 566,795 SNPs were retained for subsequent CNV calling.

\section{Copy number variations calling}

The CNVs were called using the PENNCNV software based on a hidden Markov model (HMM) and SNP chip data [22]. The PENNCNV algorithm incorporates various sets of information such as total signal intensity (Log $\mathrm{R}$ ratio [LRR]), the allelic intensity ratio (B allele frequency, $[\mathrm{BAF}]$ ), the distance between SNPs, the population frequency of B allele (PFB) simultaneously for accurate $\mathrm{CNV}$ detection. Canonical genotype clustering files were generated successively by generating affy_geno_cluster.pl and normalize_affy_geno_cluster. $\mathrm{pl}$ programs from three original chip genotype information files: AxiomGT1.call.txt, AxiomGT1.confidences.txt and AxiomGT1.summary.txt. Individual-based signal intensity (LRR) was split by kcolumn.pl program from the canonical genotype clustering files that contain the LRR values and the $\mathrm{BAF}$ values for each marker in each individual [23].

The Sscrofa genome assembly (10.2 version, ftp://ftp.en sembl.org/pub/release-89/fasta/sus_scrofa/dna/) was explored to annotate SNPs. The PFB file was created from signal intensity data, which was then explored to detect raw $\mathrm{CNV}$ calls using the default -test algorithm of the hhall.hmm model program. To minimize false-positive rate in CNV identification, we used strict quality control and filtering criteria to generate raw $\mathrm{CNV}$ calls. Individuals with a standard deviation of $L R R>0.3$, BAF drift $>0.02$, a waviness factor $>0.05$ or $<-0.05$ were filtered. Five samples were discarded during quality control, leaving us 90 animals for further analyses. Finally, the PENNCNV software was explored to identify loss (deletion) and gain (duplication) events in target regions. 
Overlapped CNVs that were detected by more than one individual in a certain region were merged to form CNVRs. According to the distribution frequencies of these CNVs in their covered region, the location of the $2.5 \%$ cumulative frequencies at both end of the region was defined as the start and end position of CNVRs.

Validation of copy number variation regions by wholegenome sequence data

Two DNA pools each comprising 12 Dongxiang spotted pigs were re-sequenced with paired-end reads of $150 \mathrm{bp}$ at $30 \times$ coverage using a Hiseq/NovaSeq platform. One pool contained DNA of 12 sows and the other included DNA of 10 sows and two boars. Reads were aligned against the Sscrofa reference genome (version 10.2) using Burrows-Wheeler Aligner (BWA) [24]. The bam file of mapped paired-end reads was sorted and indexed with marked PCR duplicates and local realignment and qualities were recalibrated. CNV caller software was then explored to detect CNVs [25]. The main steps included: i) divided the reference genome into the specified size of sliding window; ii) counted the number of read segments in the matching window; iii) absolute copy number correction to the similarity between the windows; iv) corrected and normalized GC contents; v) detected copy number based on the standardized reading signal [25]. We selected $400 \mathrm{bp}$ as a sliding window and adopted the suggested parameters.

\section{Gene contents and functional annotation in copy} number variation regions

Gene contents in CNVRs were retrieved using the genes in ensemble genes 89 database downloaded from ftp://ftp.en sembl.org/pub/release-89/gff3/sus_scrofa//Sus_scrofa.Sscrofa 10.2.89.chr.gff3.gz. We first converted the unannotated pig ensemble genes to orthologous mouse ensemble genes, then functional annotation was performed with the clueGO function in Cytoscape v3.6.0 (http://www.cytoscape.org/) for gene ontology (GO) and Kyoto encyclopedia of genes and genomes (KEGG) pathway enrichment analyses. We also compared the identified CNVRs with the reported quantitative trait loci (QTL) in the pig QTL database (http://www. animalgenome. org/cgi-bin/QTLdb/SS/index) or those CNVs reported in previous studies.

\section{RESULTS}

Genome-wide detection of copy number variations

In the present study, 90 Dongxiang spotted pigs were successfully genotyped using the Affymetrix Axiom Pig1.4M array plate. After quality control, the filtered SNPs were explored to detect CNVs using the PennCNV software. A total of $3,871 \mathrm{CNV}$ s were identified on 17 autosomes. The average number of CNVs was 43.01 per individual. By aggregating the overlapping CNVs, we detected 2,221 CNVRs on the 17 autosomes. We further eliminated the CNVRs that were detected only in one individual, leaving us 166 CNVRs. The $166 \mathrm{CNVRs}$ ranged from $2.89 \mathrm{~kb}$ to $617.53 \mathrm{~kb}$, with a mean value of $93.65 \mathrm{~kb}$ and a genome coverage of $15.55 \mathrm{Mb}$, corresponding to $0.58 \%$ of the pig genome. Of the $166 \mathrm{CNVRs}$, $111(66.87 \%)$ were identified as gain events, $46(27.71 \%)$ as loss events, and $9(5.42 \%)$ as gain-loss event (Supplementary Table S1). The 166 CNVRs were not uniformly distributed across the pig genome (Figure 1). Twenty-six (15.66\%) CNVRs were detected on Sus scrofa chromosome (SSC) 2, while no CNVR was found on SSC18. On average, 1.84 CNVRs were detected per individual.

Validation of copy number variation regions by wholegenome sequence data

Whole-genome sequence data of two DNA pools of Dongxiang spotted pigs were explored to detect CNVRs using CNVcaller [25]. We detected 700 discrete CNVRs based on the whole-genome sequence data, of which 119 perfectly overlapped with the 166 CNVRs inferred from the wholegenome 1.4 M SNP data (Supplementary Table S2).

\section{Comparison of the identified copy number variation regions with previous reports}

We compared the predicted CNVRs in this study with the reported CNVRs inferring from whole-genome chip SNP markers in 11 previous studies (Table 1). In total, 94 (56.63\%) CNVRs were consistent with previous studies. The other 72 (43.37\%) CNVRs were reported for the first time, of which 39 were validated by the whole-genome sequence data (Supplementary Table S3). The best concordance (36.75\% CNVR count, 9.52\% CNVR length) was observed between this study and the previous report by Paudel et al [26]. The second best match (21.69\% CNVR count and 7.58\% CNVR length) was found when compared to the results of Chen et al [8].

Analysis of genes content and overlapped quantitative trait loci within copy number variation regions A total of 100 genes partially or entirely spanning 77 CNVRs were retrieved from the ensemble genes 89 database, including 92 protein-coding genes, three snRNA genes, one miRNA gene, one rRNA gene and three pseudogenes (Supplementary Table S4). To better understand the functional enrichment of the 100 genes, we performed GO and KEGG pathway analyses of these genes using the clueGO function in the Cytoscape software (http://www.cytoscape.org/). We found 8 statistically significant GO terms enriched for these genes after Bonferroni correction. The significant GO terms included sensory perception of chemical stimulus or smell, detection of chemical stimulus involved in sensory perception of smell 


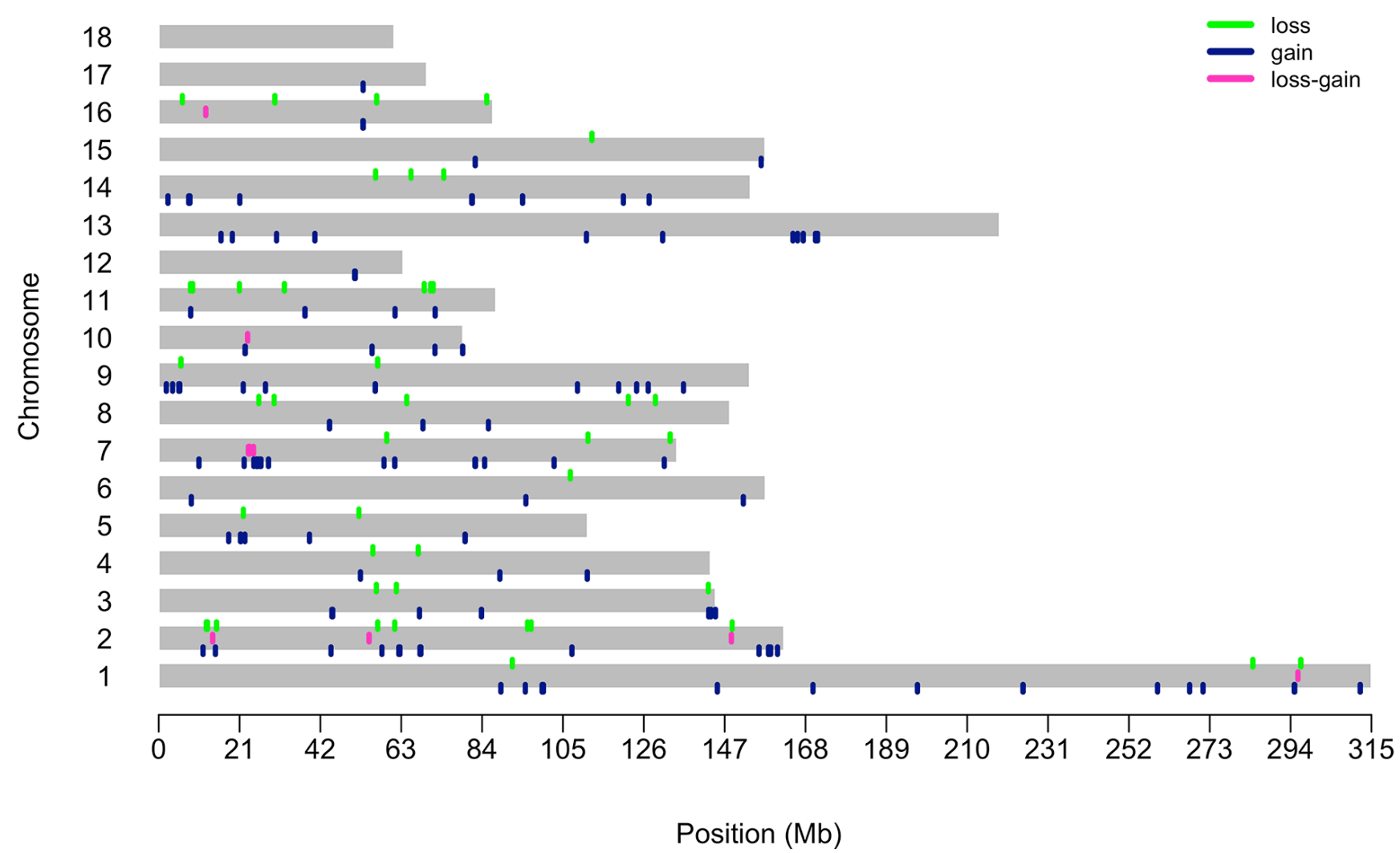

Figure 1. Distribution of copy number variation regions in the genome of Dongxiang spotted pigs. The X-axis values represent the chromosome position in Mb on the Sus scrofa 10.2 reference genome assembly. The Y-axis show all autosomal chromosomes.

and ATP hydrolysis coupled ion transmembrane transport activity (Table 2). For the KEGG analysis, CNVRs-harbored genes were significantly enriched in one pathway: olfactory transduction (Table 2). In addition, 162 (97.59\%) CNVRs were found to overlap with 2,765 previously reported QTLs affecting 378 phenotypic traits related to meat and carcass quality, reproduction, exterior, health and production, etc. (Supplementary Table S5).

\section{DISCUSSION}

We herein detected 166 CNVRs in this breed. These CNVRs covered $15.55 \mathrm{Mb}$, accounting for $0.58 \%$ of the pig autosomal genome, a proportion larger than $0.33 \%$ reported in highly inbred Iberian pigs using the 60k BeadChip SNP data [27], but within the range of $0.33 \%$ to $6.14 \%$ CNVR coverage as previously reported in other pig populations [28-30]. The different genome coverage and imperfect overlap of CNVRs among different studies may be due to several factors, such

Table 1. Comparison of CNVRs identified in this study with those 11 previous studies

\begin{tabular}{|c|c|c|c|c|c|c|}
\hline Study & Platform & Sample & CNVR & Total length (Mb) & Overlapping & Concordant length (Mb) \\
\hline Chen et al [8] & 60k SNP array & 1,693 & 565 & 139.9 & 36 & 1.18 \\
\hline Wang et al [33] & 60k SNP array & 14 & 63 & 9.98 & 6 & 0.27 \\
\hline Paudel et al [26] & NGS & 16 & 3,118 & 39.2 & 61 & 1.48 \\
\hline Wang et al [28] & 60k SNP array & 302 & 348 & 150.5 & 10 & 0.28 \\
\hline G. Schiavo et al [45] & 60k SNP array & 297 & 170 & 72.33 & 10 & 0.29 \\
\hline Fernandez et al [27] & 60k SNP array & 223 & 65 & 9.68 & 6 & 0.33 \\
\hline Dong et al [14] & 60k SNP array & 96 & 105 & 16.71 & 15 & 0.54 \\
\hline Wang et al [30] & NGS & 49 & 3,131 & 42.1 & 16 & 0.13 \\
\hline Wang et al [46] & NGS & 252 & 455 & 11.36 & 2 & 0.02 \\
\hline Xie et al [17] & 60k SNP array & 120 & 172 & 80.41 & 23 & 0.80 \\
\hline Revilla et al [29] & NGS & 7 & 540 & 9.65 & 26 & 0.35 \\
\hline
\end{tabular}

CNVRs, copy number variation regions; SNP, single nucleotide polymorphism; NGS, next-generation sequencing. 
Table 2. Go ontology (GO) and Kyoto encyclopedia of genes and genomes (KEGG) pathway analyses of genes in the identified copy number variation regions

\begin{tabular}{|c|c|c|c|c|c|}
\hline SUID & ID & Term & $p$ value & Associated gene (\%) & No. gene \\
\hline 62 & G0:0007606 & Sensory perception of chemical stimulus & $1.05 E-33$ & 6.57 & 35 \\
\hline 63 & G0:0007608 & Sensory perception of smell & $2.04 \mathrm{E}-34$ & 7.47 & 34 \\
\hline 66 & G0:0009593 & Detection of chemical stimulus & $6.32 \mathrm{E}-33$ & 6.67 & 34 \\
\hline 69 & G0:0050906 & Detection of stimulus involved in sensory perception & 7.85E-33 & 6.53 & 34 \\
\hline 73 & G0:0050907 & Detection of chemical stimulus involved in sensory perception & 5.05E-34 & 7.25 & 34 \\
\hline 81 & G0:0050911 & Detection of chemical stimulus involved in sensory perception of smell & $2.19 \mathrm{E}-35$ & 8.00 & 34 \\
\hline 89 & G0:0090662 & ATP hydrolysis coupled transmembrane transport & 3.06E-03 & 4.17 & 3 \\
\hline 90 & G0:0099131 & ATP hydrolysis coupled ion transmembrane transport & $5.42 \mathrm{E}-03$ & 4.35 & 3 \\
\hline 93 & KEGG:04740 & Olfactory transduction & $8.94 \mathrm{E}-33$ & 7.64 & 32 \\
\hline
\end{tabular}

as sample size, genetic background, $\mathrm{CNV}$ calling platforms and algorithms, and filtering criteria. In this study, we detected 72 novel CNVRs, which could be attributed to the high-density SNP markers $(n=566,795)$ with a small average distance $(3.08 \mathrm{~Kb})$ among adjacent SNPs on the customized Axiom Pig1.4M array plate.

It has been shown that $\mathrm{CNV}$ s tend to occur near telomeres or centromeres and some genomic regions are particularly apt to structural rearrangements that create CNV hotspots [31]. We herein identified two or more genes in each of 30 CNVRs, one in each of 47 CNVRs, and none in 89 CNVRs (Supplementary Table S4), suggesting that CNVs are preferably located in gene-poor regions in pigs [32]. We further showed that CNVR-related genes were significantly enriched in GO terms related to sensory perception of chemical stimulus or smell and ATP hydrolysis coupled ion transmembrane transport. These genes were also overrepresented in the olfactory transduction KEGG pathway. Our finding is consistent with previous reports that most of CNVR-overlapped genes are related to the olfactory receptors in pigs $[8,14,33]$.

A total of 162 (97.59\%) CNVRs resided in 2,765 reported QTLs affecting 378 phenotypic traits. These QTLs mainly affect several economically important traits, such as meat and carcass quality, reproduction, exterior, health and production. We note that CNVR30 overlap with a QTL for mycoplasma hyopneumoniae antibody titer. This CNVR comprises the cytochrome P450 4F2 gene that may play a key role in swine mycoplasma pneumoniae susceptibility. Mycoplasma pneumonia of swine (MPS) is a chronic and endemic respiratory disease caused by Mycoplasma hyopneumoniae and inflicts significant economic loss in swine industry [34]. There are obvious sensitivity differences among various pig breeds. For instance, compared with Western modern breeds, Chinese local pig breeds have a more intense immune response and a higher antibody level after infection with mycoplasma pneumonia [35]. Cytochrome P450 enzymes is known to mediate the suppression of inflammatory response caused by $\mathrm{Myco-}$ plasma hyopneumoniae (MPS) via peroxisome proliferator activated receptor gamma signal pathway in pigs [36]. Therefore, we assume that CNVR30 may have effects on immunity and resistance to MPS in Dongxiang spotted pigs. It is deserved to be mentioned that 72 unique CNVRs were identified in Dongxiang spotted pigs. These CNVRs correspond to 32 QTLs affecting meat and carcass quality, immunity function and reproduction traits (Supplementary Table S6). CNVR65 is located in multiple overlapped QTLs for carcass quality and harbors the thyrotropin releasing hormone degrading enzyme gene related to growth and meat production traits in sheep [37,38]. Moreover, CNVR100 overlaps with QTLs for reproduction traits including litter size, teat number and uterine capacity. Lymphoid enhancer binding factor 1 (LEF1), one gene associated with teat number [39], falls in CNVR100. LEF1 belonging to T-cell specific factor family, a small family in vertebrates including four members. As a major transcription factor of wingless signaling (Wnt), LEF1 mediates $\beta$-catenin that binds to DNA through a high conserved sequence CTTTGT [40]. LEF1 is an outstanding candidate gene for melanoma susceptibility as it can regulate microphthalmia-associated transcription factor, KIT-ligand (KITLG), tyrosinase, and other melanogenic genes [41,42]. LEF1 has be shown to associate with the development of white/black coat color in mink skin [43]. Dongxiang spotted pigs also have white/black coat colors, and we suspect that LEF1 CNVR may play a role in the formation of their coloring. Further studies are needed to test this assumption.

We are aware that there are some limits in the current study. First, a larger sample size would have enabled us to identify more CNVs. Second, SNPs in sex chromosomes were discarded. An improved algorithm is required to detect CNVs on the sex chromosomes. Finally, to reduce false-positive findings, we focused on the CNVs containing three or more consecutive SNPs (on average $3.08 \mathrm{~kb}$ ) and discarded the CNVRs that were presented in only one individual. A proportion of true low-frequency CNVs that comprise sporadic cases may have not been analyzed [44].

In summary, we used a customized Affymetrix Axiom Pig1.4M data to uncover 3,871 CNVs and 166 CNVRs from 90 Dongxiang spotted pigs. The 166 CNVRs are unevenly distributed across the pig genome. The GO and KEGG enrichment analyses illustrate that $166 \mathrm{CNVRs}$ are involved in 
a number of molecular functions especially in olfactory transduction. Of these CNVRs, 94 (56.63\%) have been reported in previous studies, whereas 72 (43.37\%) CNVRs are identified for the first time. These novel CNVRs improve the catalog of pig CNVs, which would benefit further identification of trait-related CNVs and contribute to selective breeding of Dongxiang spotted pigs and other breeds.

\section{CONFLICT OF INTEREST}

We certify that there is no conflict of interest with any financial organization regarding the material discussed in the manuscript.

\section{ACKNOWLEDGMENTS}

We thank Professor Lusheng Huang at the State Key Laboratory of Pig Genetic Improvement and Production Technology of China for his kind assistance in experimental design and management. This study was supported by National Basic Research Program (2014CB138501), Program for New Century Excellent Talents in University and Earmarked Fund for Jiangxi Agriculture Research System (JXARS-01).

\section{REFERENCES}

1. Iafrate AJ, Feuk L, Rivera MN, et al. Detection of large-scale variation in the human genome. Nat Genet 2004;36:949-51. https://doi.org/10.1038/ng1416

2. Sebat J, Lakshmi B, Troge J, et al. Large-scale copy number polymorphism in the human genome. Science 2004;305:5258. https://doi.org/10.1126/science.1098918

3. Conrad DF, Pinto D, Redon R, et al. Origins and functional impact of copy number variation in the human genome. Nature 2010;464:704-12. https://doi.org/10.1038/nature08516

4. Hou Y, Liu GE, Bickhart DM, et al. Genomic characteristics of cattle copy number variations. BMC Genomics 2011;12:127. https://doi.org/10.1186/1471-2164-12-127

5. Nicholas TJ, Cheng Z, Ventura M, Mealey K, Eichler EE, Akey JM. The genomic architecture of segmental duplications and associated copy number variants in dogs. Genome Res 2009; 19:491-9. https://doi.org/10.1101/gr.084715.108

6. Guryev V, Saar K, Adamovic T, et al. Distribution and functional impact of DNA copy number variation in the rat. Nat Genet 2008;40:538-45. https://doi.org/10.1038/ng.141

7. Doan R, Cohen N, Harrington J, et al. Identification of copy number variants in horses. Genome Res 2012;22:899-907. https://doi.org/10.1101/gr.128991.111

8. Chen C, Qiao R, Wei R, et al. A comprehensive survey of copy number variation in 18 diverse pig populations and identification of candidate copy number variable genes associated with complex traits. BMC Genomics 2012;13:733. https://doi. org/10.1186/1471-2164-13-733

9. Johansson A, Pielberg G, Andersson L, Edfors-Lilja I. Polymorphism at the porcine Dominant white/KIT locus influence coat colour and peripheral blood cell measures. Anim Genet 2005;36:288-96. https://doi.org/10.1111/j.1365-2052.2005. 01320.x

10. Wright D, Boije H, Meadows JR, et al. Copy number variation in intron 1 of SOX 5 causes the Pea-comb phenotype in chickens. PLoS Genet 2009;5:e1000512. https://doi.org/10.1371/journal. pgen.1000512

11. Rosengren Pielberg G, Golovko A, Sundstrom E, et al. A cisacting regulatory mutation causes premature hair graying and susceptibility to melanoma in the horse. Nat Genet 2008; 40:1004-9. https://doi.org/10.1038/ng.185

12. Fontanesi L, Beretti F, Riggio V, et al. Copy number variation and missense mutations of the agouti signaling protein (ASIP) gene in goat breeds with different coat colors. Cytogenet Genome Res 2009;126:333-47. https://doi.org/10.1159/000268089

13. Clop A, Vidal O, Amills M. Copy number variation in the genomes of domestic animals. Anim Genet 2012;43:503-17. https://doi.org/10.1111/j.1365-2052.2012.02317.x

14.Dong K, Pu Y, Yao N, et al. Copy number variation detection using SNP genotyping arrays in three Chinese pig breeds. Anim Genet 2015;46:101-9. https://doi.org/10.1111/age.12247

15. Fadista J, Nygaard M, Holm LE, Thomsen B, Bendixen C. A snapshot of CNVs in the pig genome. PLoS One 2008;3:e3916. https://doi.org/10.1371/journal.pone.0003916

16. Ramayo-Caldas Y, Castello A, Pena RN, et al. Copy number variation in the porcine genome inferred from a $60 \mathrm{k} \mathrm{SNP}$ BeadChip. BMC Genomics 2010;11:593. https://doi.org/10. 1186/1471-2164-11-593

17.Xie J, Li R, Li S, et al. Identification of copy number variations in Xiang and Kele pigs. PLoS One 2016;11:e0148565. https:// doi.org/10.1371/journal.pone.0148565

18. Wang X, Wang C, Huang M, et al. Genetic diversity, population structure and phylogenetic relationships of three indigenous pig breeds from Jiangxi Province, China, in a worldwide panel of pigs. Anim Genet 2018;49:275-83. https://doi.org/10.1111/ age. 12687

19. Wang C, Wang X, Tang J, et al. Genome-wide association studies for two exterior traits in Chinese Dongxiang spotted pigs. Anim Sci J 2018;89:868-75. https://doi.org/10.1111/asj.13003

20.Sambrook J, Fritsch EF, Maniatis T. Molecular cloning: a laboratory manual. Cold Spring Harbor, NY, USA: Cold Spring Harbor Laboratory Press; 1989.

21.Purcell S, Neale B, Todd-Brown K, et al. PLINK: a tool set for whole-genome association and population-based linkage analyses. Am J Hum Genet 2007;81:559-75. https://doi.org/10. 1086/519795

22. Wang K, Li M, Hadley D, et al. PennCNV: an integrated hidden Markov model designed for high-resolution copy number variation detection in whole-genome SNP genotyping data. 
Genome Res 2007;17:1665-74. https://doi.org/10.1101/gr. 6861907

23. Kendall KM, Rees E, Escott-Price V, et al. Cognitive performance among carriers of pathogenic copy number variants: analysis of 152,000 UK biobank subjects. Biol Psychiatry 2017; 82:103-10. https://doi.org/10.1016/j.biopsych.2016.08.014

24. Li H, Durbin R. Fast and accurate short read alignment with Burrows-Wheeler transform. Bioinformatics 2009;25:1754-60. https://doi.org/10.1093/bioinformatics/btp324

25. Wang X, Zheng Z, Cai Y, et al. CNVcaller: highly efficient and widely applicable software for detecting copy number variations in large populations. Gigascience 2017;6:1-12. https://doi. org/10.1093/gigascience/gix115

26. Paudel Y, Madsen O, Megens HJ, et al. Evolutionary dynamics of copy number variation in pig genomes in the context of adaptation and domestication. BMC Genomics 2013;14:449. https://doi.org/10.1186/1471-2164-14-449

27. Fernandez AI, Barragan C, Fernandez A, Rodriguez MC, Villanueva B. Copy number variants in a highly inbred Iberian porcine strain. Anim Genet 2014;45:357-66.

28. Wang Y, Tang Z, Sun Y, et al. Analysis of genome-wide copy number variations in Chinese indigenous and western pig breeds by 60 K SNP genotyping arrays. PLoS One 2014;9: e106780. https://doi.org/10.1371/journal.pone.0106780

29. Revilla M, Puig-Oliveras A, Castello A, et al. A global analysis of CNVs in swine using whole genome sequence data and association analysis with fatty acid composition and growth traits. PLoS One 2017;12:e0177014. https://doi.org/10.1371/ journal.pone.0177014

30. Wang $\mathrm{H}$, Wang $\mathrm{C}$, Yang $\mathrm{K}$, et al. Genome wide distributions and functional characterization of copy number variations between Chinese and Western pigs. PLoS One 2015;10:e0131522. https://doi.org/10.1371/journal.pone.0131522

31. Itsara A, Cooper GM, Baker C, et al. Population analysis of large copy number variants and hotspots of human genetic disease. Am J Hum Genet 2009;84:148-61. https://doi.org/10. 1016/j.ajhg.2008.12.014

32. Freeman JL, Perry GH, Feuk L, et al. Copy number variation: new insights in genome diversity. Genome Res 2006;16:94961. https://doi.org/10.1101/gr.3677206

33. Wang J, Wang H, Jiang J, et al. Identification of genome-wide copy number variations among diverse pig breeds using SNP genotyping arrays. PLoS One 2013;8:e68683. https:/doi.org/ 10.1371/journal.pone.0068683

34. Simionatto S, Marchioro SB, Maes D, Dellagostin OA. Mycoplasma hyopneumoniae: from disease to vaccine development. Vet Microbiol 2013;165:234-42. https://doi.org/10.1016/j. vetmic.2013.04.019

35. Fang XM, Zhao WM, Fu YF, et al. Difference in susceptibility to mycoplasma pneumonia among various pig breeds and its molecular genetic basis. Scientia Agricultura Sinica 2015; 48:2839-47. https://doi.org/10.3864/j.issn.0578-1752.2015. 14.015

36. Fang X, Zhao W, Xu J, et al. CYP1A1 mediates the suppression of major inflammatory cytokines in pulmonary alveolar macrophage (PAM) cell lines caused by Mycoplasma hyponeumoniae. Dev Comp Immunol 2016;65:132-8. https://doi.org/10.1016/ j.dci.2016.06.023

37.Zhang L, Liu J, Zhao F, et al. Genome-wide association studies for growth and meat production traits in sheep. PLoS One 2013;8:e66569. https://doi.org/10.1371/journal.pone.0066569

38.Zhang L, Ma X, Xuan J, et al. Identification of $M E F 2 B$ and TRHDE gene polymorphisms related to growth traits in a new Ujumqin sheep population. PLoS One 2016;11:e0159504. https://doi.org/10.1371/journal.pone.0159504

39.Xu RX, Wei N, Wang Y, Wang GQ, Yang GS, Pang WJ. Association of novel polymorphisms in lymphoid enhancer binding factor 1 (LEF-1) gene with number of teats in different breeds of pig. Asian-Australas J Anim Sci 2014;27:1254-62. https:// doi.org/10.5713/ajas.2013.13772

40.Xie X, Lu J, Kulbokas EJ, et al. Systematic discovery of regulatory motifs in human promoters and 3' UTRs by comparison of several mammals. Nature 2005;434:338-45. https://doi.org/10. 1038/nature03441

41. Guenther CA, Tasic B, Luo L, Bedell MA, Kingsley DM. A molecular basis for classic blond hair color in Europeans. Nat Genet 2014;46:748-52. https://doi.org/10.1038/ng.2991

42. Villareal MO, Kume S, Neffati M, Isoda H. Upregulation of Mitf by phenolic compounds-rich Cymbopogon schoenanthus treatment promotes melanogenesis in B16 melanoma cells and human epidermal melanocytes. Biomed Res Int 2017; 2017:Article ID 8303671. https://doi.org/10.1155/2017/8303671

43.Song X, Xu C, Liu Z, et al. Comparative transcriptome analysis of mink (Neovison vison) skin reveals the key genes involved in the melanogenesis of black and white coat colour. Sci Rep 2017;7:12461. https://doi.org/10.1038/s41598-017-12754-0

44. Redon R, Ishikawa S, Fitch KR, et al. Global variation in copy number in the human genome. Nature 2006;444:444-54. https://doi.org/10.1038/nature05329

45.Schiavo G, Dolezal MA, Scotti E, et al. Copy number variants in Italian Large White pigs detected using high-density single nucleotide polymorphisms and their association with back fat thickness. Anim Genet 2014;45:745-9. https://doi.org/10. 1111/age. 12180

46. Wang Z, Chen Q, Liao R, et al. Genome-wide genetic variation discovery in Chinese Taihu pig breeds using next generation sequencing. Anim Genet 2017;48:38-47. https://doi.org/10.1111/ age. 12465 